\title{
La calidad alimentaria en una economía cognitiva: el caso de la denominación de origen vitivinícola "León"*
}

Food Quality in a Cognitive Economy:

The Case of the Wine Designation of Origin "León"

A qualidade alimenticia em uma economia cognitiva:

o caso da denominação de origem vitivinicola "León"

Alfredo Macías Vázquez**

Recibido: 12 de junio del 2019

Aprobado: 28 de abril del 2020

https://doi.org/10.12804/revistas.urosario.edu.co/territorios/a.7998

Para citar este artículo

Macías Vázquez, A. (2021). La calidad alimentaria en una economía cognitiva: el caso de la denominación de origen vinícola "León". Territorios, (44), 293-312. https://doi.org/10.12804/revistas.urosario.edu. co/territorios/a.7998

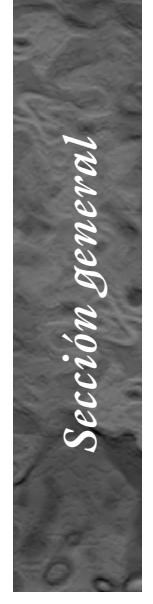

\footnotetext{
* Este trabajo ha sido posible en el marco de las Ayudas a Proyectos de $I+D+i$ correspondientes al Programa Estatal de Investigación, Desarrollo e Innovación orientada a los Retos de la Sociedad del Ministerio de Economía, Industria y Competitividad, Gobierno de España. Ref. CSO2017-85188-R.

** Universidad de León, León (España). Correo electrónico:amacv@unileon.es. ORCID: https:// orcid.org/0002-26842666.
} 
Palabras clave

Calidad; conocimiento; origen; valor; vino.

Keywords Knowledge; origin; quality; value; wine.

Palavras-chave Qualidade; conbecimento; valor; origem; vinho.

tersitarias 44

\section{RESUMEN}

El modelo español de certificaciones geográficas de calidad alimentaria muestra signos de agotamiento. Los cambios recientes cuestionan su función como mecanismo para proteger las rentas territoriales, obligando a analizar las prácticas de los actores en la cadena de valor con nuevos enfoques. Centrados en el análisis de la Denominación de Origen Protegida "León”, el objetivo de este trabajo es interrogar los determinantes de la generación de valor en una economía posindustrial. Se constató la importancia central de ciertas mediaciones entre la producción y el consumo que sistematizan, conectan, interpretan y validan los conocimientos locales, las cuales presentan un comportamiento deficiente que incide de manera negativa en la mejora de la calidad y en la valorización de los vinos.

\section{ABSTRAC}

The Spanish model of geographical food quality certifications shows signs of exhaustion. The recent changes question its function as a mechanism to protect territorial rents, forcing to analyze the practices of the actors in the value chain with new approaches. Focusing on the analysis of the Protected Designation of Origin "León", the objective of this work is to interrogate about the determinants of the generation of value in a post-industrial economy. The central importance of certain mediations between production and consumption, that systematize, connect, interpret and validate local knowledge was verified, which present a deficient behaviour that negatively affects the improvement of the quality and the value of the wines.

\section{RESUMO}

O modelo espanhol de certificações geográficas de qualidade alimentícia mostra sinais de esgotamento. Mudanças recentes questionam sua função como mecanismo para proteção das rendas territoriais, obrigando-nos a analisar as práticas dos atores da cadeia de valor sob novos enfoques. Nos concentramos na análise da denominação de origem protegida "León", nosso objetivo é questionarmo-nos sobre os determinantes da geração de valor em uma economia pós-industrial. Constatamos a importância central de certas mediações entre a produção e o consumo, que sistematizam, conectam, interpretam e validam os conhecimentos locais. As mesmas, apresentam, um comportamento deficiente que incide negativamente na melhora da qualidade e da valorização dos vinhos. 


\section{Introducción}

El modelo español de calidad alimentaria basado en certificaciones territoriales, en particular, denominaciones de origen, muestra síntomas de agotamiento. Si bien este modelo contribuyó de forma significativa, aunque con resultados dispares, a un reposicionamiento más ventajoso de los productores locales en la cadena de valor y a la búsqueda de una senda de desarrollo rural más inclusiva y sustentable -en especial, en aquellos territorios marginados en los precios previos y acelerados de modernización agraria-, los cambios económicos, sociales y culturales de los últimos años están cuestionando su función institucional y jurídica, como mecanismo para proteger las rentas territoriales en la producción de alimentos depositarios de una cierta identidad local. Este cuestionamiento se viene detectando en la literatura académica (Grunert \& Aachmann, 2016; Teil, 2017; Marie-Vivien et al., 2017) $\mathrm{y}$ en los propios actores y organismos internacionales como la Unión Europea (Lucatelli, 2000).

Aunque el presente trabajo se centra de manera analítica en el impacto de las transformaciones contemporáneas, esto no quiere decir que los problemas del modelo de calidad hayan comenzado recientemente, ni siquiera que dichas transformaciones sean la única causa. Existen problemáticas en el contexto institucional español, que generan incentivos para un comportamiento perverso en los agentes implicados en las producciones rurales. Las autoridades españolas, como resultado del "neoliberalismo corporativo" (Alonso González \& Macías Vázquez, 2014), han sido muy permisivas a la hora de otorgar certificaciones de calidad a determinadas producciones locales, lo cual ha provocado un exceso de denominaciones de origen e indicaciones geográficas protegidas. En el caso del vino, por ejemplo, existe una gran cantidad de denominaciones de origen en las cuales resulta complicado justificar su diferenciación, en cuanto a la singularidad varietal de sus viñedos. Ante esta situación, los consumidores experimentan una creciente dificultad para optimizar sus decisiones de consumo que, en paralelo, desincentiva a los productores a mejorar la calidad de sus alimentos. Como la información sobre la calidad de los alimentos mantiene un carácter asimétrico entre productores y consumidores, es habitual que se produzcan situaciones de selección adversa cuando esta dificultad para reconocer la calidad de los caldos se traduce en un descenso de la disposición a pagar por parte de los demandantes.

Por lo general, es difícil salir de estas dinámicas. Incluso, se puede llegar a producir la desaparición o reducción significativa de ciertos mercados, como ocurrió en el caso de la Denominación de Origen "Vino de Jerez" (Fernández, 2010). De por sí, estas dinámicas trágicas, tanto a nivel social como ambiental, ya justificarían un análisis pormenorizado de su naturaleza, su evolución y sus posibles 
soluciones. Sin embargo, hoy en día, las dificultades que este modelo de calidad tiene para cumplir su función como herramienta para la protección de las rentas territoriales, tiene su origen en procesos estructurales de mayor calado. De hecho, es difícil explicar los cambios que se están produciendo en el mercado con meros estudios que hagan referencia al comportamiento del consumidor (Parga Dans et al., 2019). Se están produciendo fenómenos como la crisis generalizada de rentabilidad entre los productores españoles -O portugueses - de vino a precios bajos, o la irrupción de los vinos de terroir que, vendiendo vino a precios elevadísimos, recuperan el dinamismo económico de regiones enteras, que difícilmente pueden explicarse sin incorporar en el análisis los cambios estructurales asociados al desarrollo de economías posindustriales. De este modo, surgen nuevos etiquetados - producción orgánica, biodinámica, vino natural-, o se introducen nuevas dimensiones de la calidad de los alimentos - sobre todo, los relacionados con el impacto en la salud de sus componentes nutricionales-, que hacen necesario avanzar hacia un análisis más complejo de las prácticas de producción y de diferenciación puestas en marcha por los actores implicados en la cadena de valor.

Con el desarrollo de una economía basada en el conocimiento, se hace necesario identificar, explorar y analizar

\section{territarias 44} nuevas formas de innovación social rural que permitan generar sinergias positivas en la cadena de valor $y$, alternativas para construir socialmente la calidad y la identidad territorial de los alimentos, y así, incorporar en el análisis una interacción más compleja entre las dimensiones materiales e inmateriales de los procesos de valorización a escala territorial (Belletti et al., 2017). En los últimos años, denominaciones de origen como "Rías Baixas", que en apariencia despuntaba como referente en los vinos blancos de calidad con valor añadido, luchan por sobrevivir en el segmento de los vinos de bajo precio, compitiendo con denominaciones como "Rueda" o "Ribeiro". Sin embargo, a poca distancia de las Rías Baixas, un vino tinto de la Denominación de Origen "Bierzo", La Faraona (2015), supera los 1170 USD por botella.

Dicho lo anterior, el objetivo de este texto es indagar sobre los determinantes de este proceso, en qué medida un modelo de calidad alimentaria se ve condicionado por las nuevas formas de generación de valor asociadas con el desarrollo de la economía postindustrial, qué nuevos valores, significados y atributos se vinculan con la calidad de los alimentos en una economía basada en el conocimiento $\mathrm{y}$, por último, cuáles son las mediaciones cognitivas que están surgiendo entre la producción y el consumo, cómo y qué actores las ponen en marcha. Dar respuesta a estas preguntas implicaría ubicar el debate sobre la construcción de la calidad alimentaria en un 
plano completamente diferente, lo que contribuye a un rediseño de las políticas públicas que fomentan el desarrollo rural.

\section{Las mediaciones cognitivas y su relevancia para el desarrollo rural}

En el contexto económico actual, el conocimiento se ha erigido como una variable estructural en los procesos de generación de valor, tan importante como comprender los procesos productivos "materiales" que se gestan en la comunidad local, por lo que resulta importante analizar la dimensión inmaterial de los procesos de valorización y el papel de los diferentes actores de la cadena de valor en dichos procesos. Para aproximarse a esta dimensión y al rol de los actores implicados en ella, lo rural debe comprenderse como una combinación compleja de procesos productivos, relaciones de poder, representaciones, discursos y entramados institucionales variados (Cloke, 2006; Dewsbury, 2003). Distintas configuraciones de conocimiento, poder y distribución del valor, definen los modos de interacción entre actores extraterritoriales y actores locales. Siguiendo trabajos de la "economía política de la cultura" (Jessop \& Oosterlynck, 2008; Sayer, 2001), se considera fundamental para entender las sociedades rurales contemporáneas, la combinación del interés de la economía política en cuestiones como el valor, el intercambio, la distribución y el poder en el mundo rural (Jones, 2008), con elementos del giro cultural centrados en la construcción social de los valores, el conocimiento, la cultura (Cloke, 1997) y, su asociación a los aparatos estatales y los "circuitos culturales del capital" (Thrift, 2005).

Del mismo modo, la "aproximación económica cultural" del desarrollo rural (Ray, 1998) enfatiza en el intento por localizar el control económico de las comunidades como respuesta a presiones extraterritoriales exacerbadas por el neoliberalismo, que promueve una concepción 'emprendedora' según la cual, todos los territorios tienen oportunidades económicas al disponer de diferencias culturales valorizables en el mercado, incluyendo lenguajes, saber-haceres, patrimonios, paisajes o comidas y alimentos tradicionales. Es decir, frente a la concepción neoliberal del territorio, se hace necesario tomar en consideración las variables y los instrumentos que permiten desarrollar la capacidad territorial de apropiación de valor en una economía basada en el conocimiento, pues los territorios no son meras mercancías que se reinventan de modo permanente mientras se fortalece la lógica depredadora y rentista de los capitales locales y extraterritoriales (Marazzi, 2008; Vercellone, 2008). En este sentido, es importante comprender cómo se genera valor en una economía basada en el conocimiento, pero también es decisivo identificar aquellos eslabones críticos y vulnerabilidades en los cuales los actores locales suelen mostrar 
más dificultades a la hora de apropiarse territorialmente del valor generado en dichas economías.

Para abordar estas cuestiones, se trabajó con un modelo de generación de valor a partir de la producción de conocimiento $(E)$ que deriva del ensamblaje de tres drivers (Rullani, 2004a): la capacidad de interpretación de los significados incorporados en la producción material de alimentos $(\mathcal{v})$ por parte de los consumidores, la cantidad de veces que se propagan y replican estos significados $(n) \mathrm{y}$, la capacidad de apropiación del valor generado en los distintos actores que participan en su producción y sostenimiento $\left(p_{i}\right.$, donde $\left.0 \leq p_{\mathrm{i}} \leq 1\right)$, dicho modelo se formula mediante la siguiente ecuación:

$$
E=v \cdot n \cdot p_{i}
$$

Al tomar como referencia este modelo, las investigaciones realizadas en casos anteriores advierten sobre varias circunstancias preocupantes en la evolución de las denominaciones de origen españolas (Macías Vázquez \& Alonso González, 2015). Por un lado, en denominaciones de origen de aceite de oliva y vino, se pone en evidencia que la ausencia de innovaciones organizativas a nivel colectivo les impide apropiarse $\left(p_{i}\right)$ del valor generado como resultado de la implantación de los signos de calidad, que, paradójicamente, ha mejorado la percepción de los consumidores en cuanto a la calidad del producto territarias 44 298 conocimientos y prácticas locales a través de una exitosa estrategia de codificación en los mercados $(n)$. Como resultado de esta incapacidad para regular y apropiarse de la producción de valor, los productores locales se han visto forzados a intensificar todavía más los cultivos para mantener sus niveles de renta, lo cual ha tenido consecuencias negativas a nivel medioambiental y en la propia calidad y seguridad alimentaria - erosión y envenenamiento de los suelos, residuos químicos en los alimentos, pérdida de biodiversidad, entre otras-. En buena medida, estas dinámicas trágicas señalan que la transición hacia economías del conocimiento cuestionan las formas tradicionales de certificación geográfica de la calidad, al no dar cuenta de los aspectos relacionales del conocimiento y concebir producción y consumo de forma estanca y aislada (Chiffoleau, 2009). De hecho, la producción y el uso del conocimiento son actividades intrínsecamente sociales que implican la generación de significados, valores, símbolos y sentidos que adquieren valor solo si otros los comparten y se los apropian. Por ello, la economía del conocimiento es una economía de conexiones y de flujos en permanente retroalimentación, en la que resulta imposible separar la producción y el consumo en la medida en que este último también implica una actividad de producción cognitiva (Goodman, 2016).

Por otro lado, las nuevas tecnologías de la información y la comunicación se han constituido en un potente mediador 
para articular la propagación de significados, valores y conocimientos en redes e interacciones a distancia, no limitadas al ámbito local. Pero, en paralelo, esta mayor capacidad de propagación cognitiva $(n)$ ha puesto de manifiesto una menor capacidad de regulación de la producción de valor $\left(p_{i}\right)$ por parte de aquellas estructuras tradicionales de acción colectiva, organizadas a escala local. En buena medida, porque esta mayor capacidad de propagación ha implicado la necesidad de explorar nuevos contextos sociales, en concreto, de comprender los significados, los valores y los deseos que están detrás de las prácticas de consumo alimentario - que influyen de forma directa en "v"-, lo cual conlleva plantearse los problemas de la acción colectiva en una escala más amplia, con unos contenidos y mediaciones diferentes.

En dichas circunstancias, el problema que se plantea en líneas generales es el siguiente: cómo transformar un conocimiento original desarrollado en un contexto $\alpha$, en un conocimiento conectivo que permita su reutilización en diferentes contextos $\beta$, teniendo en cuenta que $\alpha$ y $\beta$ son contextos diferentes, con actores, recursos, un medio ambiente y un tejido de relaciones sociales específicas (Rullani, 2004 b). La integración del conocimiento, en este sentido, no puede abordarse como un proceso lineal, automático y mecánico, dada la naturaleza específica de los recursos locales. Por ello, es necesario articular una serie de mediadores cuya función sea fortalecer la integración de los conocimientos, de tal forma que se pueda minimizar el riesgo de fragmentación (Rullani, 2004b). De manera esquemática, se clasificaron estos mediadores en la siguiente tipología de operadores, llamados "mediadores cognitivos":

- Sistematizadores: ensamblan la producción de conocimientos de cada especialista particular en un determinado contexto territorial, suelen tomar la forma de asociaciones de productores, asociaciones de desarrollo territorial, agencias de inteligencia comarcal, entre otras.

- Conectores: vinculan conocimientos generados en contextos diversos, toman la forma de redes, plataformas, encuentros periódicos o jornadas puntuales.

- Intérpretes: recontextualizan los conocimientos originales para que puedan ser utilizados en contextos diferentes, trabajan sobre los deseos del consumidor, toman la forma de mercados alternativos, museos, organizaciones de consumidores, centros de dietética $\mathrm{o}$, incluso, de agencias de viaje.

- Validadores: intervienen en relación con la incertidumbre en la validez de uso del conocimiento en los diferentes contextos, pueden basarse en el desarrollo de la participación y de las relaciones de confianza (sistemas participativos de calidad) o en 
la construcción de indicadores objetivos (certificaciones territoriales de calidad).

De aquí la necesidad de analizar las nuevas formas de acción colectiva en una sociedad posindustrial, entendida como espacio de intercambio cultural y simbólico (Hassanein, 2003). La acción colectiva no puede comprenderse desde una lógica basada en la elección racional individual o grupal, sino que resulta necesario explorar lo que la Teoría del Actor-Red denomina como procesos de co-construcción de nuevos valores y significados en las interacciones sociales en el ámbito alimentario (Kaljonen, 2006). En concreto, en lugar de dar por hecho la calidad, la autenticidad o el valor de los alimentos, el objetivo será explorar su construcción social por parte de una cadena extensa de actores que generan nuevos valores y significados, además de nuevas identidades y movimientos sociales que, basados en la acción colectiva, producen innovaciones y transformaciones relacionales generadoras de desarrollo endógeno y territorial (Starr, 2010; Friedmann \& Macnair, 2008).

\section{Metodología de la investigación}

Este trabajo pretende realizar una aportación metodológica que contribuya a un mejor análisis de la construcción social de la calidad de los alimentos en un determinado territorio, a partir de una reconsideración de las relaciones entre el mundo de la producción y del consumo en una sociedad posindustrial. La mayoría de investigaciones parte de la existencia de terroir y calidad, dando por hecho lo que ha de ser investigado. Así, las investigaciones existentes dejan sin resolver la cuestión de si el terroir y la calidad son objetivas en el origen, el clima y la naturaleza, como asumen las certificaciones territoriales de calidad; o subjetivas, es decir, mediadas por un número amplio de actores y sus relaciones. Por tal razón, se hace necesario analizar la construcción social, simbólica y material de la calidad de un alimento, más allá del marketing y la distinción mediante el consumo (Bourdieu, 1984). Desde esta perspectiva, la construcción social de la calidad emerge como un conjunto de realidades simbólicas con consecuencias materiales, generadas en un ámbito de discursos y prácticas interdependientes alrededor de la producción y consumo del producto. Explorar esta dinámica implica, a nivel metodológico, el empleo de la técnica de "seguir a los actores" (Latour, 2013).

Para abordar estas cuestiones, el análisis se centra en la Denominación de Origen (D.O.) “León", situada en la provincia española de idéntica denominación (figura 1). Creada en el 2007, la D.O. "León" lucha por ganar notoriedad en un marco completamente diferente al de las denominaciones vitivinícolas que se crearon el siglo pasado. Así mismo, el interés de este caso de estudio viene marcado por las diferentes estrategias seguidas por las dos 
Figura 1. D.O. “León” en las denominaciones españolas

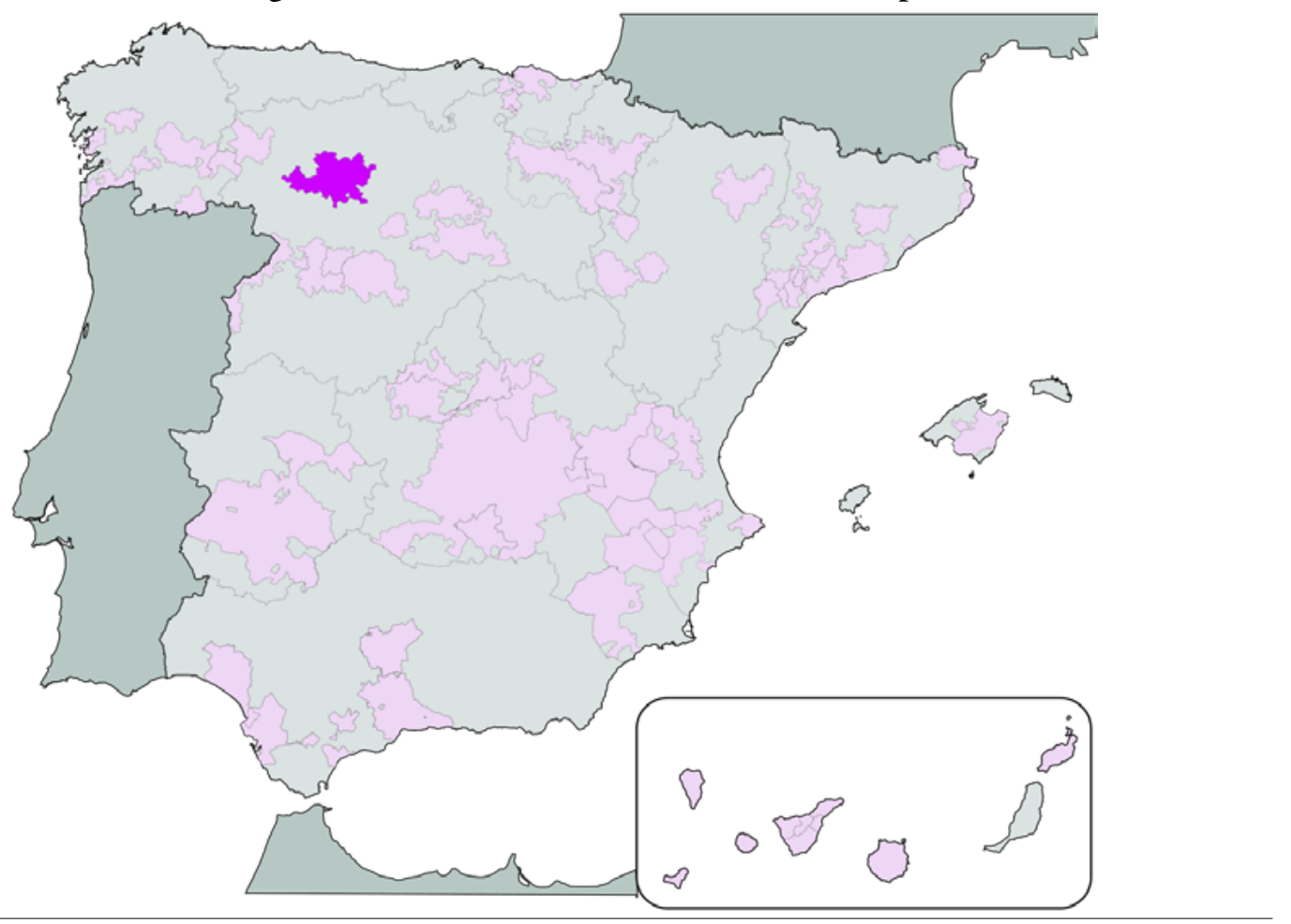

Fuente: Wikimedia Commons (2009).

subzonas de esta denominación analizadas en este trabajo, a saber, Valdevimbre y Los Oteros, y cómo sus resultados vienen determinados por la adecuación de esas estrategias a las nuevas condiciones impuestas por la generación de valor en una economía posindustrial. Se realizaron 18 entrevistas semiestructuradas y libres a agentes relacionados con esta denominación: gerentes de bodega, representantes del Consejo Regulador de la D.O. "León", investigadores del Instituto de la Viña y el Vino de la Universidad de León, periodistas especializados y responsables de la administración provincial. También se utilizaron fuentes documentales de las organizaciones profesionales, noticias de prensa, informes gubernamentales y legislación, que ayudaron a construir un contexto de interpretación.

\section{Los vinos leoneses y la} problemática de su valorización

Entre los primeros documentos del Reino de León que datan del siglo $\mathrm{X}$, ya se hacía 
- El Páramo Bajo, otra comarca que ya no es productora, ha sufrido una completa sustitución del viñedo viejo por los cultivos de regadio.

\section{territarias 44} 302 referencia a la importancia de los viñedos en la economía del sur de León (Revilla, 2016). Bajo el auspicio de los principales monasterios de la zona, los vinos leoneses llegaron a proveer a buena parte del norte peninsular en aquella época. Con el paso del tiempo, la producción de vino se redujo, otros cultivos tomaron el relevo y aparecieron zonas vitivinícolas más dinámicas, que alcanzaron una notoriedad mayor. Lo más característico son las bodegas-cuevas rupestres, espacios excavados en pequeñas lomas o promontorios de tierra arcillosa aprovechando el desnivel para formar galerías. A pesar de la larga historia de los vinos leoneses, se produjo un deterioro de la reputación de estos caldos, que pasaron a considerarse un vino elaborado a nivel familiar, con escaso conocimiento enológico y de una calidad muy inferior a otros vinos del noroeste peninsular.

En el 2007, se constituyó legalmente la Denominación de Origen "Tierra de León" - que recientemente se denominó solo "León"-, después de un largo periplo de intentos frustrados. Sus tres principales comarcas productoras son Valdevimbre, Los Oteros y la Ribera del Cea. La situación de partida era lamentable, ya que en décadas pasadas muchos viticultores habían vendido sus derechos de plantación de viñedos a bodegas de las D.O. "Bierzo" y "Ribera de Duero", produciéndose un arranque masivo de cepas viejas, en particular en las comarcas de Los Oteros y de la Ribera del Cea. ${ }^{1} \mathrm{Si}$ bien resultaba complicado dinamizar la zona, al menos la constitución de la D.O. ha representado una oportunidad para frenar el proceso de abandono de la viticultura en el lugar. Según datos de la propia D.O., la superficie cultivada de viñedo asciende a $3317 \mathrm{~km}^{2}$, siendo cuarenta bodegas las inscritas en la D.O. En comparación con otras denominaciones próximas, la producción es pequeña aunque creciente, a pesar de que las heladas tardías del 2017 representaron una reducción dramática de la producción. En las mejores cosechas, se han llegado a recoger 4500 t de uva. De una comercialización total de 2,4 millones de botellas, 2,0 millones se venden con la certificación de calidad otorgada por el Consejo Regulador ubicado en Valencia de Don Juan, al sureste de la provincia leonesa. Desde el 2007, casi todos los años, las cosechas han sido calificadas como "muy buenas", o incluso, en el 2014 y 2015 como "excelentes".

El mencionado retraso en la constitución legal de la D.O., si se compara con las denominaciones vitivinícolas más cercanas geográficamente de la región de Castilla y León, que han conseguido un cierto grado de notoriedad en el mercado - "Bierzo", “Toro" o "Ribera de Duero"-, es subrayado por la mayoría de las personas entrevistadas como una desventaja relevante a la hora de enfrentarse a los desafíos de un mercado tan competitivo y dinámico como el vitivinícola. Lo paradójico es que los vinos leoneses, además de tener una historia centenaria, presentan una 
tipicidad mucho más marcada que otros vinos con más notoriedad de la región, tanto a nivel de la viticultura como en el proceso de vinificación.

En el caso de la viticultura, la D.O. "León" ostenta como uva principal el prieto picudo, que ocupa prácticamente el $70 \%$ de la superficie cultivada con viñedos. Mientras que en otras denominaciones con mayor notoriedad, las variedades de uva suelen representar una modificación de otra más general — como ocurre con el tempranillo-, en el caso de la mencionada variedad leonesa, es específica del territorio, siendo muy difícil encontrar una especie semejante en alguna otra parte del mundo. Además, hay que tener en cuenta que las propiedades que le da al vino esta variedad de uva (acidez, astringencia), son claramente distinguibles para el consumidor. En el caso de la vinificación, el prieto picudo ha persistido con técnicas ancestrales en la elaboración de vinos, que le otorgan una singularidad elevada en el contexto de los vinos españoles. En específico, la técnica del "madreo" 2 da lugar a vinos rosados con una peculiar "aguja", un contenido carbónico perceptible al paladar en vinos jóvenes, que de nuevo, se hace distinguible por parte del consumidor.

En realidad, se habla de un vino único en el mundo. No obstante, otras especificidades del proceso productivo merman la posibilidad de rentabilizar los caldos, en especial cuando se cultivan viñedos antiguos. En este caso, hay que aplicar la "poda en rastra", que junto a la vigorosidad de las cepas de pietro picudo, hace la tarea más laboriosa y compleja que en otras variedades. Por otro lado, Margón, una bodega ubicada en Pajares de los Oteros, que apuesta por el vino de terroir a partir de viñas viejas y rastreras, señala que la mecanización de la vendimia en este tipo de viñas es imposible, mientras que un trabajador en un viñedo antiguo puede recoger entre 150 y $180 \mathrm{~kg} /$ día, en un viñedo nuevo en espaldera puede llegar a vendimiar $1000 \mathrm{~kg} /$ día. Sin embargo, como se analizará más adelante, la apuesta por los vinos de terroir a partir del cultivo de parcelas de viñedo viejo, no es el vector de valorización más dinámico en esta denominación.

Más allá de estos inconvenientes, el principal problema de los vinos leoneses es el escaso valor agregado que genera una producción tan típica y singular. Así, si el precio medio de los vinos de la región castellana y leonesa gira en torno a los 125 USD/hl, en la provincia leonesa oscila en torno a los 60 USD/hl (Consejería de Agricultura y Ganadería, 2017). En realidad, la baja valorización de los vinos comienza en el proceso de recogida de la uva. Como reconocen los directivos de la D.O. "León", la uva no se paga bien si se compara con otras denominaciones de origen. En un año normal, el kilogramo de uva se paga en torno a los 35-40 céntimos de USD, lo que en muchos casos impide cubrir los costes de producción en la viticultura
${ }^{2}$ En el pliego de condiciones de la D.O. se especifica que la técnica del madreo consiste en la adicción por parte del bodeguero o del enólogo, de racimos enteros $y$ sanos de la variedad de uva pietro picudo a los depósitos de vino rosado durante la fermentación. La cantidad de racimos añadidos debe oscilar entre el 5 o $10 \%$ de la capacidad del depósito donde se realiza la fermentación. El momento en que se produce la adicción de esta cantidad de racimos dependerá del criterio del enólogo, pudiendo ser al inicio de la fermentación, antes de que la densidad baje de los 1000 gramos por centímetro cúbico, o bien, se pueden ir añadiendo pequeñas cantidades de racimos antes de completar la cifra ya indicada, en tres momentos diferentes de la fermentación. Una vez finalizada la fermentación, los racimos adicionados al depósito se desechan.

tersitarios 44

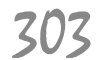


y pone en riesgo la conservación de las técnicas tradicionales.

De otro lado, se mantiene una estrategia conservadora en el ámbito de la comercialización, apoyada incluso por los directivos del Consejo Regulador, que contrasta con la "revolución comercial" llevada adelante por las denominaciones de origen en muchas zonas rurales españolas históricamente marginadas (Sanz Cañada \& Macías Vázquez, 2005). Tradicionalmente, los mercados finales del prieto picudo han sido León y Asturias, y no parece que la política comercial de la D.O. vaya más allá de la mera defensa de estos mercados cercanos. De hecho, es escasa la labor de promoción que el Consejo Regulador realiza más allá de la organización de las ferias de la D.O. en León y Valencia de Don Juan, a las cuales asisten cada vez menos bodegas inscritas en la propia denominación. Las únicas actividades realizadas a nivel nacional, tal como señalan en la propia D.O., son las presentaciones de vinos en algunas ferias y eventos: Alimentaria (Barcelona), Gourmet (Madrid) y Fenavin (Ciudad Real), que son ferias dirigidas a importadores, prensa especializada y hostelería. En consecuencia, no existe casi ninguna actividad promocional dirigida de manera directa a los consumidores finales, fuera de los mercados tradicionales.

De manera polémica, en una de las bodegas más dinámicas en términos de comercialización y marketing, Leyenda del Páramo, se denuncia la falta de ambición teniendo en cuenta la calidad de los caldos y, se señala que la D.O. funciona bajo un esquema obsoleto. Por ejemplo, se comenta que resulta desfasado continuar reduciendo la actividad del Consejo Regulador a una actividad administrativa de control de la calidad, cuando hoy en día los mercados - sobre todo los externos- exigen el cumplimiento de unos parámetros mucho más rigurosos para los vinos de calidad. En su opinión, el Consejo Regulador debería centrar su actividad en el ámbito de la promoción y la comercialización, poniendo en valor las variedades autóctonas como el prieto picudo y el albarín. En ese sentido, un investigador del Instituto de la Viña y el Vino de la Universidad de León, incide en el mismo aspecto, pone de relieve cómo el abandono de una estrategia más audaz ha impedido aprovechar el crecimiento del mercado para los vinos blancos jóvenes, a partir del albarín - debido a los cambios en las preferencias de los consumidores en los mercados anglosajones y europeos-, tal como hicieron en su momento otras variedades de uva blanca, como el albariño (Macías Vázquez \& Vence Deza, 2013).

\section{Una denominación, dos mundos paralelos}

Según el último Informe Nielsen (2018), en el cual se analiza la evolución del mercado de vino español durante el 2017, a pesar de la escasa promoción, los vinos rosados de la D.O. "León” — producidos con 
la técnica del "madreo"-, se consolidan en el cuarto puesto del consumo nacional de este tipo de vinos, con un $5,4 \%$ de la cuota de mercado. En el resto de los vinos, la cuota de mercado es completamente marginal. Aunque no todos los proyectos que pretenden romper con la dinámica de parálisis que impera en la denominación, logran obtener resultados prometedores. Por ejemplo, Bodegas Margón, ha apostado por los vinos de terroir utilizando viñedos con cepas de más de 100 años, bajo el asesoramiento del que ha sido premiado como mejor enólogo del mundo en el 2016, el leonés Raúl Pérez. Margón realiza de forma manual toda la vendimia y no usa herbicidas, por lo cual las viñas viejas y rastreras adquieren un aspecto "selvático". La intención es elaborar un vino lo más natural posible, en el sentido que exprese las particularidades de cada parcela en toda su riqueza. De hecho, Bodegas Margón ha comenzado una política comercial basada en el embotellamiento del vino por parcelas: Valdemuz, elaborado con las viñas más viejas y Paraje del Santo, que resulta de la unión de varias parcelas con suelo arenoso, lo cual ha dado lugar a caldos muy diferentes entre sí, a pesar de utilizar los mismos procesos de vinificación.

Sin embargo, Margón reconoce que está siendo muy complicado subir el precio de las botellas, no quedando más remedio que dedicar buena parte de la producción de uva a la elaboración de vinos algo inferiores, a un precio menor. En realidad, la dificultad para poner en marcha vinos de terroir no obedece exclusivamente a un problema de intensa competencia entre las bodegas de diferentes denominaciones. Además, se están produciendo disfunciones importantes entre las mediaciones cognitivas que vinculan el mundo de la producción y el consumo. En este caso, se puede observar cómo la hostelería española - a diferencia de lo que ocurre en otros mercados - no cumple su función interpretativa, de cara a mejorar la capacidad de valorización de los vinos por parte del consumidor. Como apuntan varios bodegueros de la D.O. "León" en las entrevistas realizadas, lo que ocurre es que el hostelero busca vinos de marcas y denominaciones que logren una elevada rotación de las existencias. De esta forma, todas las botellas que superan los 23 USD ni siquiera se incorporan en la carta de vinos de muchos restaurantes.

La Bodega Margón, que encabeza uno de los proyectos más innovadores de la comarca de Los Oteros, es muy pesimista respecto al futuro de dicho territorio. Por un lado, señala la alta potencialidad de valorización de estas tierras, que se alcanzan a considerar de los mejores lugares de España para elaborar vinos naturales. Los viñedos pueden llegar a encontrarse a 850 metros de altitud, en una zona hiperventilada y con un diferencial térmico significativo entre el día y la noche, que impide que las bacterias asociadas a determinadas plagas tengan tiempo de reproducirse y propagarse. 
De hecho, por muchos años no hay que realizar ningún tratamiento fitosanitario. Por otro lado, Margón considera que la ausencia de importantes inversiones empresariales en la D.O. "León”, como sí ha ocurrido en las D.O. "Ribera del Duero", "Bierzo" o "Rioja", ha impedido la consolidación de ciertos liderazgos que empujen el desarrollo territorial. En su opinión, si este tipo de liderazgos hubiesen existido, no se habría producido el arranque masivo de cepas viejas como resultado de la concentración parcelaria, ni tampoco la venta de derechos de plantación a bodegas de otras denominaciones. Ha sido tal la destrucción del patrimonio vitícola local, que ya resulta impensable que una bodega foránea de prestigio pudiese realizar una inversión en la zona. Aunque reconoce que, en parte, esta situación representa una oportunidad para los viticultores locales, es consciente de la dificultad para activar un proceso orientado al fortalecimiento de la reputación de los vinos de la comarca.

Otra opción competitiva serían los vinos naturales o ecológicos. De manera pionera, la Bodega Leyenda del Páramo ha sacado en los últimos años una marca de vino ecológico, El Rescatado. En este caso, lo curioso es que la producción se destina en principio a los mercados externos, de hecho, la apuesta competitiva de esta bodega es reconvertir sus viñedos a la viticultura ecológica y llegar a exportar el $80 \%$ de la producción, en la actualidad exportan el $20 \%$. Aunque reconoce que el consumidor español no busca en el lineal de la distribución, ni demanda en la restauración a los vinos ecológicos; observa, no obstante, que este tipo de vinos se está convirtiendo en una clase con más dinamismo de mercado y con unas posibilidades mayores de agregación de valor a nivel internacional, dado que el consumidor de vino en los países desarrollados cada vez se interesa más en los caldos ecológicos. En esta bodega, que nos recibieron tras su regreso de la feria asiática de vinos, celebrada en Singapur, son enormemente críticos con el esquema de funcionamiento de las denominaciones de origen en España. En la actualidad, Leyenda del Páramo, que probablemente sea una de las bodegas más activas en la búsqueda de mercados alternativos a los tradicionales - junto con Pardevalles, que exporta actualmente el $40 \%$ de su producción-, cuenta con catorce vinos diferentes: blancos, rosados, tintos, ecológicos, dulces, espumosos, entre otros. Sin embargo, solo cinco de ellos se encuentran calificados por la D.O. "León", aunque dada su orientación al mercado internacional, la etiqueta de la denominación les resulta cada vez menos relevante. En su opinión, la rigidez del reglamento de la D.O. se está convirtiendo en un obstáculo para que las bodegas puedan competir en los mercados, en especial, en los externos. Por ejemplo, la elaboración de vinos dulces o espumosos es prácticamente imposible dentro del marco regulatorio de la D.O.

Leyenda del Páramo y Pardevalles, están ubicadas en la comarca de Valdevimbre, 
que se ha convertido en la zona de producción más dinámica de la denominación, tanto en términos productivos como $\mathrm{CO}^{-}$ merciales. Su evolución contrasta con lo que está ocurriendo en Los Oteros, gracias a la combinación de otros vectores materiales e inmateriales de valorización - como la gastronomía, el enoturismo o el patrimonio - con la producción vitivinícola. Valdevimbre tiene una serie de recursos específicos, cuyo potencial de valorización ha sido bien gestionado por determinados mediadores cognitivos entre la producción y el consumo, tales como agencias de viajes, hosteleros, museos o autoridades locales. Una peculiaridad muy marcada de esta comarca, que la convierte en un caso único, consiste en que, al menos desde el siglo XVI (Revilla, 2016), sus bodegas se encontraban ubicadas en cuevas. En los últimos años, estas cuevas han sido reconvertidas en restaurantes, con un éxito creciente de público. Así, cada fin de semana Valdevimbre recibe una media de 2000 comensales, procedentes de núcleos urbanos importantes como Madrid o Valladolid. Estos visitantes, además de comer en las cuevas-restaurantes con vinos de la D.O. "León" — de la comarca de Valdevimbre-, realizan actividades de enoturismo y visitas al Museo del Vino del Ayuntamiento de Valdevimbre y a las bodegas de la zona, donde a su vez, hacen compras de nuevas botellas de vino local.

Como se ha observado, la comarca de Valdevimbre ha iniciado una senda de desarrollo vinculada al mundo del vino.
Se puede decir, incluso, que la comarca se ha transformado en un auténtico “distrito vitivinícola". Este logro no ha sido el resultado de la implementación de la D.O., ni de la acción deliberada de sus representantes, ni de las autoridades locales. Menos aún ha sido el producto de las fuerzas espontáneas del mercado y su lógica competitiva. Tampoco puede decirse que esta experiencia de desarrollo rural haya sido el resultado mecánico de la adopción de tecnologías de la información y la comunicación. En realidad, ha sido la consecuencia de una combinación virtuosa de la acción de toda una serie de mediadores cognitivos que, en especial, en el plano de la interpretación, la sistematización y la conexión de los conocimientos locales, ha conseguido relacionar e integrar en una misma cadena de valor a los productores locales (de un contexto $\alpha$ ) con los consumidores (de otros contextos $\beta$ ). Esta integración se ha realizado a través de mediaciones completamente diferentes a las existentes en los mercados tradicionales, como puede ser la gran distribución moderna (Sanz Cañada, 2002). De hecho, puede afirmarse que representa un caso muy singular en el mundo español del vino, en relación directa con los procesos de generación de valor en una economía basada en el conocimiento.

\section{Conclusiones}

Los resultados de esta investigación confirman la idoneidad del planteamiento territarias 44

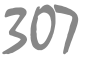


metodológico enunciado. Como se plantea desde la sociología pragmática francesa o la "sociología del gusto" (Hennion, 2004), la aproximación de este trabajo a la construcción de la calidad alimentaria como un proceso socialmente dinámico (Teil, 2011), mejora en gran medida la capacidad para abordar de manera conceptual los procesos de desarrollo rural en una economía basada en el conocimiento. Este tipo de aproximación difiere de las concepciones tradicionales de la calidad, tanto de tipo objetivista - que, desde las ciencias exactas, analizan el vino y otros alimentos con base en parámetros físicoquímicos - como de carácter subjetivista -que, como se ha mencionado en la introducción, consideran el problema desde la perspectiva unilateral del consumidor individual y del marketing-.

A partir de estas concepciones tradicionales, el problema fundamental es que los productores rurales han accedido al mercado mediatizados por un componente abstracto y socialmente construido, que implicaba una determinada lógica para lograr cierto estatus y notoriedad de los alimentos de calidad y, de esta manera, aumentar su precio. Lo que se ha intentado en este trabajo es, precisamente, cuestionar estos enfoques y plantear que, con el desarrollo de economías posindustriales se abren nuevas oportunidades de mediación entre el mundo de la producción y el consumo, siempre y cuando se comprenda a cabalidad su lógica de funcionamiento.
Para llegar a esta conclusión, se intentó "seguir a los actores" y, de esta manera, comprobar lo inadecuado que resulta preguntarse por las consecuencias económicas de la reducción del funcionamiento del mercado a una lógica de significados abstractos que llegan a través de los alimentos (Tonkiss, 2006). Por esta vía, se termina excluyendo la realidad productiva, institucional y social del sector de los alimentos de calidad (Overton \& Murray, 2013), excluyendo del análisis la complejidad de las prácticas productivas y de la relación entre valor y gusto (Allen \& Germov, 2011). Como se pudo observar en el análisis del estudio de caso, esas prácticas y relaciones condicionan el proceso de diferenciación de la producción a partir de los imaginarios de calidad existentes, ofreciendo la posibilidad de aumentar el valor generado por el vino mediante estrategias colectivas de apropiación del valor simbólico o inmaterial.

Desde esta perspectiva, la investigación muestra cómo la construcción social de la calidad alimentaria emerge como un conjunto de realidades simbólicas con consecuencias materiales, generado en un ámbito de discursos y prácticas interdependientes alrededor del mundo de la producción y del consumo, y, de las conexiones entre ambos en una economía posindustrial. La comparación entre las dos subzonas de la D.O. "León” analizadas en este trabajo, Los Oteros y Valdevimbre, permite comprender cómo se plasman - $\mathrm{O}$ 
fracasan-estos procesos en las realidades concretas, poniendo de manifiesto que los esquemas actuales para entender los procesos de construcción social de la calidad alimentaria resultan obsoletos.

El análisis del estudio de caso indica que es necesario repensar la noción de acción colectiva en una economía basada en el conocimiento. Se hace importante dejar atrás las nociones basadas en criterios de elección racional, para vincular el fenómeno de la acción colectiva con los reiterados procesos de resignificación de la producción y el consumo en una economía cognitiva, poniendo de relieve la importancia de construir una acción colectiva que transcienda los contextos locales. En este sentido, las estructuras de gobernanza asociadas con las certificaciones territoriales de calidad resultan progresivamente inapropiadas para comprender y gestionar las dinámicas estructurales, al generar, no solo crecientes asimetrías de información y problemas de selección adversa, sino también restricciones institucionales a la iniciativa de los actores rurales - tal como se observa en el caso de la Bodega Leyenda del Páramo-. De manera alternativa, la acción colectiva debe transcender el ámbito territorial, asumiendo que la gestión de recursos inmateriales impone una mayor incidencia y un mayor conocimiento de los mercados lejanos, de los contextos no locales. Para ello, es necesario incorporar en los esquemas de acción colectiva y en las propias estructuras territoriales de gobernanza a los mediadores cognitivos que, desde el desarrollo de funciones de sistematización, conexión, interpretación y validación - lo que permite incorporar, a su vez, a las denominaciones de origen-, realizan tareas esenciales en la generación de valor y en su apropiación territorial, en una economía basada en el conocimiento.

\section{Referencias}

Allen, M. P., \& Germov, J. (2011). Judging taste and creating value: The cultural consecration of Australian wines. Journal of Sociology, 47(1), 35-51. https:// doi.org/10.1177/1440783310380988

Alonso González, P., \& Macías Vázquez, A. (2014). Neoliberalismo corporativo y clientelismo en España: etnografía de la financiación europea del desarrollo rural a través de un proyecto fallido. AIBR: Revista de Antropologia Iberoamericana, 9(3), 223-250. https://dialnet.unirioja.es/servlet/ articulo? $\operatorname{codigo}=4947056$

Belletti, G., Marescotti, A., \& Touzard, J. M. (2017). Geographical indications, public goods, and sustainable development: The roles of actors' strategies and public policies. World Development, 98, 45-57. https://doi.org/10.1016/j. worlddev.2015.05.004

Bourdieu, P. (1984). Distinction: A social critique of the judgement of taste. Harvard University Press.

Chiffoleau, Y. (2009). From politics to co-operation: The dynamics of embeddedness in alternative food supply territarias 44 309 


\section{territarias 44}

chains. Sociologia Ruralis, 49(3), 218-235. https://doi.org/10.1111/ j.1467-9523.2009.00491.x

Cloke, P. (1997). Country backwater to virtual village? Rural studies and the cultural turn'. Journal of Rural Studies, 13(4), 367-375. https://doi. org/10.1016/S0743-0167(97)000533

Cloke, P. (2006). Conceptualizing rurality. En P. J. Cloke, T. Marsden \& P. H. Mooney (Eds.), Handbook of rural studies (pp. 18-28). SAGE.

Consejería de Agricultura y Ganadería (2017). Anuario de estadística agraria de Castilla y León. Junta de Castilla y León.

Dewsbury, J. D. (2003). Witnessing space: Knowledge without contemplation. Environment and Planning A, 35(11), 1907-1932. https://doi. org/10.1068\%2Fa3582

Fernández, E. (2010). Unsuccessful responses to quality uncertainty: Brands in Spain's sherry industry, 1920-1990. Business History, 52(1), 100-119. https://doi. org/10.1080/00076790903469638

Friedmann, H., \& Mcnair, A. (2008). Whose rules rule? Contested projects to certify 'local production for distant consumers'. Journal of Agrarian Change, 8(2-3), 408-434. https://doi.org/10.1111/j.14710366.2008.00175.x

Goodman, M. K. (2016). Food geographies I: Relational foodscapes and the busy-ness of being more-than-food. Progress in Human Geography, 40(2), 257-266. https:// doi.org/10.1177/0309132515570192

Grunert, K. G., \& Aachmann, K. (2016). Consumer reactions to the use of $\mathrm{EU}$ quality labels on food products: A review of the literature. Food Control, 59, 178-187. https://doi.org/10.1016/j. foodcont.2015.05.021

Hassanein, N. (2003). Practicing food democracy: a pragmatic politics of transformation. Journal of Rural Studies, 19(1), 77-86. https://doi. org/10.1016/S0743-0167(02)000414

Hennion, A. (2004). Pragmatics of taste. En M. Jacobs \& N. Hanrahan (Eds.), The Blackwell companion to the sociology of culture (pp. 131-144). Blackwell.

Jessop, B., \& Oosterlynck, S. (2008). Cultural political economy: On making the cultural turn without falling into soft economic sociology. Geoforum, 39(3), 1155-1169. https://doi.org/10.1016/j.geoforum.2006.12.008

Jones, M. (2008). Recovering a sense of political economy. Political geography, 27(4), 377-399. https://doi. org/10.1016/j.polgeo.2008.03.003

Kaljonen, M. (2006). Co-construction of agency and environmental management. The case of agri-environmental policy implementation at Finnish farms. Journal of Rural Studies, 22(2), 205-216. https://doi.org/10.1016/j. jrurstud.2005.08.010 
Latour, B. (2013). An inquiry into modes of existence: an anthropology of the moderns. Harvard University Press.

Lucatelli, S. (2000). Appellations of origin and geographical indications in OECD member countries: economic and legal implications. En OCDE, Directorate for food, agriculture and fisheries, trade directorate (pp. 301-317). OCDE.

Macías Vázquez, A., \& Vence Deza, X. (2013). Las Denominaciones de Origen en la orientación competitiva de los procesos de innovación de los sistemas vitivinícolas locales. Revista Galega de Economía, 22(1), 97-124. https://revistas.usc.gal/index.php/rge/article/ view/1271

Macías Vázquez, A., \& Alonso González, P. (2015). Collective symbolic capital and sustainability: Governing fishing communities in a knowledge economy. Marine Policy, 53, 21-26. https://doi. org/10.1016/j.marpol.2014.11.014

Marazzi, C. (2008). Capital and language: From the new economy to the war economy. Semiotext(E).

Marie-Vivien, D., Bérard, L., Boutonnet, J. P., \& Casabianca, F. (2017). Are French geographical indications losing their soul? Analyzing recent developments in the governance of the link to the origin in France. World Development, 98, 25-34. https://doi. org/10.1016/j.worlddev.2015.01.001

Overton, J., \& Murray, W. E. (2013). Class in a glass: Capital, neoliberalism and social space in the global wine industry. Antipode, 45(3), 702-718. https://doi.org/10.1111/j.14678330.2012.01042.x

Parga Dans, E., Alonso González, P., \& Macías Vázquez, A. (2019). Taste and knowledge: The social construction of quality in the organic wine market. Human Ecology. An Interdisciplinary Journal, 47(1), 135-143. https://doi. org/10.1007/s10745-019-0051-1

Ray, C. (1998). Culture, intellectual property and territorial rural development. Sociologia Ruralis, 38(1), 3-20. https://doi.org/10.1111/14679523.00060

Revilla, J. (2016). De la cepa a la mesa: estudios históricos en torno al vino. Universidad de León.

Rullani, E. (2004a). Economia della conoscenza: Creatività e valore nel capitalismo delle reti. Carocci.

Rullani, E. (2004b). La fabbrica dell'immateriale. Produrre valore con la conoscenza. Carocci.

Sanz Cañada, J. (2002). El sistema agroalimentario español: estrategias competitivas frente a un modelo de demanda en un contexto de mercados imperfectos. En C. Gómez \& J. J. González (Coords.), Agricultura y sociedad en el cambio de siglo (pp. 143179). Mc-Graw Hill.

Sanz Cañada, J., \& Macías Vázquez, A. (2005). Quality certification, institutions and innovation in local agro-food systems: protected designations of origin of olive oil in Spain. Journal territarias 44 
of Rural Studies, 21(4), 475-486. https://doi.org/10.1016/j.jrurstud.2005.10.001

Sayer, A. (2001). For a critical cultural political economy. Antipode, 33(4), 687-708. https://doi.org/10.1111/14678330.00206

Starr, A. (2010). Local food: a social movement?. Cultural Studies $\leftrightarrow$ Critical Methodologies, 10(6), 479-490. https:// doi.org/10.1177/1532708610372769

Teil, G. (2011). No such thing as terroir? Objectivities and the regimes of existence of objects. Science, Technology, \& Human Values, 37(5), 478-505. https:// doi.org/10.1177/0162243911423843

Teil, G. (2017). Protecting appellations of origin: One hundred years of efforts and debates. En W. Van Caenegem \& J. Cleary (Eds.), The Importance of Place: Geographical Indications as a Tool for Local and Regional Development (pp. 147-171). Springer.

Thrift, N. J. (2005). Knowing capitalism. SAGE.

Tonkiss, F. (2006). Contemporary economic sociology: globalization, production, inequality. Routledge.

Vercellone, C. (2008). The new articulation of wages, rent and profit in cognitive capitalism. Queen Mary University.

Wikimedia Commons. (2009). Tierra de León (DO). https://en.wikipedia.org/ wiki/Tierra_de_Le\%C3\%B3n_(DO). 